
in $\mathrm{e}^{+} \mathrm{e}^{-}$interactions at LEP

\title{
L3 Collaboration
}

M. Acciarri ${ }^{\mathrm{z}}$, P. Achard ${ }^{\mathrm{s}}$, O. Adriani ${ }^{\mathrm{p}}$, M. Aguilar-Benitez ${ }^{\mathrm{y}}$, J. Alcaraz ${ }^{\mathrm{y}}$, G. Alemanni v, J. Allaby ${ }^{\mathrm{q}}$, A. Aloisio ${ }^{\mathrm{ab}}$, M.G. Alviggi ${ }^{\mathrm{ab}}$, G. Ambrosi ${ }^{\mathrm{s}}$, H. Anderhub au , V.P. Andreev f,aj, T. Angelescu ' , F. Anselmo i, A. Arefiev aa, T. Azemoon ${ }^{\text {c }}$, T. Aziz ${ }^{\text {j }, ~ P . ~ B a g n a i a ~}{ }^{\text {ai }}$, L. Baksay ${ }^{\text {ap }}$, A. Balandras ${ }^{\text {d }}$, R.C. Ball ${ }^{\text {c }}$, S. Banerjee ${ }^{j}$, Sw. Banerjee ${ }^{j}$, A. Barczyk ${ }^{\text {au,as }}$, R. Barillère ${ }^{q}$, L. Barone ${ }^{\text {ai }}$,

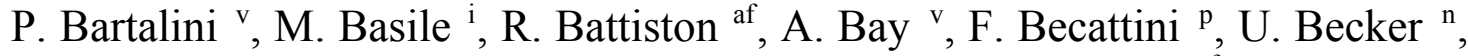
F. Behner ${ }^{\text {au }}$, L. Bellucci ${ }^{\mathrm{p}}$, J. Berdugo ${ }^{\mathrm{y}}$, P. Berges ${ }^{\mathrm{n}}$, B. Bertucci ${ }^{\text {af }}$, B.L. Betev ${ }^{\mathrm{au}}$, S. Bhattacharya ${ }^{\mathrm{j}}$, M. Biasini af ${ }^{\text {af }}$ A. Biland ${ }^{\text {au }}$, J.J. Blaising ${ }^{\mathrm{d}}$, S.C. Blyth ${ }^{\mathrm{ag}}$, G.J. Bobbink ${ }^{\text {b }}$, A. Böhm ${ }^{\text {a }}$, L. Boldizsar ${ }^{\text {m }}$, B. Borgia ${ }^{\text {ai }}$, D. Bourilkov ${ }^{\text {au }}$, M. Bourquin ${ }^{\text {s }, ~ S . ~ B r a c c i n i ~}{ }^{\text {s, J.G. Branson }}{ }^{\text {al }}$, V. Brigljevic ${ }^{\text {au }}$, F. Brochu ${ }^{\text {d, }}$, A. Buffini ${ }^{\text {p }}$, A. Buijs ${ }^{\text {aq }}$, J.D. Burger ${ }^{n}$, W.J. Burger ${ }^{\text {af }}$, J. Busenitz ${ }^{\text {ap }}$, A. Button ${ }^{c}$, X.D. Cai ${ }^{\text {n, M. Campanelli }}{ }^{\text {au }}$, M. Capell ${ }^{\text {n }}$, G. Cara Romeo ${ }^{\mathrm{i}}$, G. Carlino ${ }^{\mathrm{ab}}$, A.M. Cartacci ${ }^{\text {p }}$, J. Casaus ${ }^{y}$, G. Castellini ${ }^{\mathrm{p}}$, F. Cavallari ai, N. Cavallo ${ }^{\text {ab }}$, C. Cecchi ${ }^{\text {s }}$, M. Cerrada ${ }^{y}$, F. Cesaroni ${ }^{\text {w }}$, M. Chamizo ${ }^{\text {s, Y.H. Chang }}{ }^{\text {aw }}$, U.K. Chaturvedi ${ }^{\mathrm{r}}$, M. Chemarin ${ }^{\mathrm{x}}$, A. Chen ${ }^{\text {aw }}$, G. Chen ${ }^{\mathrm{g}}$, G.M. Chen ${ }^{\mathrm{g}}$, H.F. Chen ${ }^{\mathrm{t}}$, H.S. Chen ${ }^{\mathrm{g}}$, G. Chiefari ${ }^{\text {ab }}$, L. Cifarelli ${ }^{\text {ak }}$, F. Cindolo ${ }^{\mathrm{i}}$, C. Civinini ${ }^{\mathrm{p}}$, I. Clare ${ }^{\mathrm{n}}$, R. Clare ${ }^{\mathrm{n}}$, G. Coignet ${ }^{\mathrm{d}}$, A.P. Colijn ${ }^{\mathrm{b}}$, N. Colino ${ }^{\mathrm{y}}$, S. Costantini ${ }^{\mathrm{h}}$, F. Cotorobai ${ }^{1}$, B. Cozzoni i , B. de la Cruz ${ }^{\mathrm{y}}$, A. Csilling ${ }^{\mathrm{m}}$, S. Cucciarelli af, T.S. Dai ${ }^{\mathrm{n}}$, J.A. van Dalen ${ }^{\text {ad }}$, R. D’Alessandro ${ }^{\mathrm{p}}$, R. de Asmundis ${ }^{\mathrm{ab}}$, P. Déglon ${ }^{\mathrm{s}}$, A. Degré ${ }^{d}, K$. Deiters ${ }^{\text {as }}$, D. della Volpe ${ }^{\text {ab }}$, P. Denes ${ }^{\text {ah }}$, F. DeNotaristefani ${ }^{\text {ai }}$, A. De Salvo ${ }^{\text {au }}$, M. Diemoz ${ }^{\text {ai }}$, D. van Dierendonck ${ }^{\text {b }}$, F. Di Lodovico ${ }^{\text {au}}$, C. Dionisi ${ }^{\text {ai }}$, M. Dittmar ${ }^{\text {au }}$, A. Dominguez ${ }^{\text {al }}$, A. Doria ${ }^{\text {ab }}$, M.T. Dova ${ }^{\text {r,1 }}$, D. Duchesneau ${ }^{d}$, D. Dufournaud d, P. Duinker ${ }^{\text {b }}$, I. Duran ${ }^{\text {am }}$, H. El Mamouni ${ }^{\mathrm{x}}$, A. Engler ${ }^{\text {ag }}$, F.J. Eppling ${ }^{n}$, F.C. Erné ${ }^{\text {b }}$, P. Extermann ${ }^{\text {s }}$, M. Fabre ${ }^{\text {as }}$, R. Faccini ${ }^{\text {ai }}$, M.A. Falagan ${ }^{\mathrm{y}}$, S. Falciano ${ }^{\text {ai, }}$, A. Favara ${ }^{\mathrm{q}}$, J. Fay ${ }^{\mathrm{x}}$, O. Fedin ${ }^{\text {aj }}$, M. Felcini au, T. Ferguson ${ }^{\text {ag }}$, F. Ferroni ${ }^{\text {ai }}$, H. Fesefeldt ${ }^{\text {a }}$, E. Fiandrini ${ }^{\text {af }}$, J.H. Field ${ }^{\text {s }}$, F. Filthaut ${ }^{\mathrm{q}}$, P.H. Fisher ${ }^{\mathrm{n}}$, I. Fisk ${ }^{\text {al }}$, G. Forconi ${ }^{\text {n, }}$ 
L. Fredj ${ }^{\text {s }}$, K. Freudenreich ${ }^{\text {au }}$, C. Furetta ${ }^{\text {z }}$, Yu. Galaktionov aa,n, S.N. Ganguli j,

P. Garcia-Abia ${ }^{\text {e }}$, M. Gataullin ${ }^{\text {ae }}$, S.S. Gau ${ }^{\text {k }}$, S. Gentile ${ }^{\text {ai, }}$, N. Gheordanescu ${ }^{1}$, S. Giagu ai, Z.F. Gong ${ }^{\mathrm{t}}$, G. Grenier ${ }^{\mathrm{x}}$, O. Grimm ${ }^{\text {au }}$, M.W. Gruenewald ${ }^{\mathrm{h}}$, M. Guida ${ }^{\text {ak }}$, R. van Gulik ${ }^{\text {b }, ~ V . K . ~ G u p t a ~}{ }^{\text {ah }}$, A. Gurtu ${ }^{\text {j }, ~ L . J . ~ G u t a y ~}{ }^{\text {ar }}$, D. Haas ${ }^{\text {e }}$, A. Hasan ${ }^{\text {ac }}$, D. Hatzifotiadou i, T. Hebbeker ${ }^{\text {h}}$, A. Hervé ${ }^{\mathrm{q}}$, P. Hidas ${ }^{\mathrm{m}}$, J. Hirschfelder ${ }^{\text {ag }}$, H. Hofer ${ }^{\text {au}, ~ G . ~ H o l z n e r ~}{ }^{\text {au }}$, H. Hoorani ${ }^{\text {ag }}$, S.R. Hou ${ }^{\text {aw }}$, I. Iashvili at, B.N. Jin ${ }^{\mathrm{g}}$, L.W. Jones ${ }^{\mathrm{c}}$, P. de Jong ${ }^{\mathrm{b}}$, I. Josa-Mutuberría ${ }^{\mathrm{y}}$, R.A. Khan ${ }^{\text {r }}$, D. Kamrad ${ }^{\text {at }}$, M. Kaur ${ }^{\text {r,2 }}$, M.N. Kienzle-Focacci ${ }^{\text {s, D. Kim }}{ }^{\text {ai }}$, D.H. Kim ${ }^{\text {ao }}$, J.K. Kim ${ }^{\text {ao }}$, S.C. Kim ${ }^{\text {ao }}$, J. Kirkby ${ }^{\text {q }}$, D. Kiss ${ }^{\text {m }}$, W. Kittel ${ }^{\text {ad }}$, A. Klimentov ${ }^{\text {n,aa }}$, A.C. König ${ }^{\text {ad }}$, A. Kopp ${ }^{\text {at }}$, V. Koutsenko ${ }^{\text {n,aa }}$, M. Kräber ${ }^{\text {au }}$, R.W. Kraemer ${ }^{\text {ag }}$, W. Krenz ${ }^{\text {a }}$, A. Kunin ${ }^{\text {n,aa }}$, P. Ladron de Guevara ${ }^{\text {y }}$, I. Laktineh ${ }^{\mathrm{x}}$, G. Landi ${ }^{\mathrm{p}}, \mathrm{K}$. Lassila-Perini ${ }^{\text {au }}$, P. Laurikainen ${ }^{\text {u }}$, M. Lebeau ${ }^{\mathrm{q}}$, A. Lebedev ${ }^{\mathrm{n}}$, P. Lebrun ${ }^{x}$, P. Lecomte ${ }^{\text {au }}$, P. Lecoq ${ }^{\mathrm{q}}$, P. Le Coultre ${ }^{\mathrm{au}}$, H.J. Lee ${ }^{\mathrm{h}}$, J.M. Le Goff ${ }^{\mathrm{q}}$, R. Leiste ${ }^{\text {at }}$, E. Leonardi ai, P. Levtchenko ${ }^{\text {aj }}$, C. Li ${ }^{~}$, C.H. Lin ${ }^{\text {aw }}$, W.T. Lin ${ }^{\text {aw }}$, F.L. Linde ${ }^{b}$, L. Lista ${ }^{\text {ab }}$, Z.A. Liu ${ }^{\text {g }}$, W. Lohmann ${ }^{\text {at }}$, E. Longo ${ }^{\text {ai }}$, Y.S. Lu ${ }^{\text {g }}$, K. Lübelsmeyer ${ }^{\text {a }}$, C. Luci q,ai, D. Luckey ${ }^{n}$, L. Lugnier ${ }^{x}$, L. Luminari ai, W. Lustermann ${ }^{\text {au }}$, W.G. Ma ${ }^{\mathrm{t}}$, M. Maity ${ }^{\mathrm{j}}$, L. Malgeri ${ }^{\mathrm{q}}$, A. Malinin ${ }^{\mathrm{a}, \mathrm{q}}$, C. Maña ${ }^{\mathrm{y}}$, D. Mangeol ${ }^{\text {ad }}$, P. Marchesini au, G. Marian ${ }^{\circ}$, J.P. Martin ${ }^{\mathrm{x}}$, F. Marzano ${ }^{\text {ai }}$, G.G.G. Massaro ${ }^{b}$, K. Mazumdar ${ }^{\text {j, R.R. McNeil }}{ }^{\text {f }}$, S. Mele ${ }^{\mathrm{q}}$, L. Merola ${ }^{\mathrm{ab}}$, M. Meschini ${ }^{\mathrm{p}}$, W.J. Metzger ${ }^{\text {ad }}$, M. von der Mey ${ }^{\mathrm{a}}$, A. Mihul ${ }^{1}$, H. Milcent ${ }^{\mathrm{q}}$, G. Mirabelli ai , J. Mnich q, G.B. Mohanty ${ }^{\text {j, P. Molnar }}{ }^{\text {h }}$, B. Monteleoni p, 3 ,

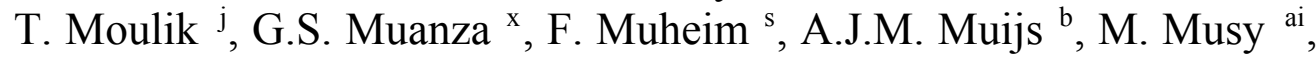
M. Napolitano ${ }^{\text {ab }}$, F. Nessi-Tedaldi ${ }^{\text {au }}$, H. Newman ${ }^{\text {ae }}$, T. Niessen ${ }^{\text {a }}$, A. Nisati ai, H. Nowak ${ }^{\text {at }}$, Y.D. Oh ${ }^{\text {ao }}$, G. Organtini ai, R. Ostonen ${ }^{\text {u }, ~ A . ~ O u l i a n o v ~}{ }^{\text {aa }}$, C. Palomares ${ }^{y}$, D. Pandoulas ${ }^{\text {a }}$, S. Paoletti ${ }^{\text {ai, }}$, P. Paolucci ${ }^{\text {ab }}$, R. Paramatti ${ }^{\text {ai }}$, H.K. Park ${ }^{\text {ag }}$, I.H. Park ${ }^{\text {ao }}$, G. Pascale ${ }^{\text {ai }}$, G. Passaleva ${ }^{\text {q }}$, S. Patricelli ${ }^{\text {ab }}$, T. Paul ${ }^{\text {k, }}$, M. Pauluzzi af ${ }^{\text {, C. Paus }}{ }^{\mathrm{q}}$, F. Pauss au ${ }^{\text {a }}$ D. Peach ${ }^{\mathrm{q}}$, M. Pedace ${ }^{\text {ai }}$, S. Pensotti ${ }^{\mathrm{z}}$, D. Perret-Gallix ${ }^{d}$, B. Petersen ${ }^{\text {ad }}$, D. Piccolo ${ }^{a b}$, F. Pierella ${ }^{i}$, M. Pieri ${ }^{p}$, P.A. Piroué ah ${ }^{\text {a }}$ E. Pistolesi ${ }^{\text {z }}$, V. Plyaskin ${ }^{\text {aa }}$, M. Pohl au ${ }^{\text {a }}$ V. Pojidaev ${ }^{\text {aa, }}$, H. Postema ${ }^{\text {n }}$, J. Pothier ${ }^{\text {q }}$, N. Produit ${ }^{\text {s }, ~ D . O . ~ P r o k o f i e v ~}{ }^{\text {ar }}$, D. Prokofiev aj, J. Quartieri ${ }^{\text {ak }}$, G. Rahal-Callot ${ }^{\text {au, }, ~}$, M.A. Rahaman ${ }^{j}$, P. Raics ${ }^{\circ}$, N. Raja ${ }^{j}$, R. Ramelli ${ }^{\text {au }}$, P.G. Rancoita ${ }^{\text {z }}$, G. Raven ${ }^{\text {al }}$, P. Razis ${ }^{\text {ac }}$, D. Ren ${ }^{\text {au }}$, M. Rescigno ai, S. Reucroft ${ }^{\text {k }}$, T. van Rhee ${ }^{\text {aq }}$, S. Riemann ${ }^{\text {at }}$, K. Riles ${ }^{\text {c }}$, A. Robohm ${ }^{\text {au }}$, J. Rodin ${ }^{\text {ap }}$, B.P. Roe ${ }^{\mathrm{c}}$, L. Romero ${ }^{\mathrm{y}}$, A. Rosca ${ }^{\mathrm{h}}$, S. Rosier-Lees ${ }^{\text {d }}$, J.A. Rubio ${ }^{\text {q }}$, D. Ruschmeier ${ }^{\text {h }}$, H. Rykaczewski ${ }^{\text {au }}$, S. Saremi ${ }^{\text {f }}$, S. Sarkar ${ }^{\text {ai }}$, J. Salicio ${ }^{\text {, }}$ E. Sanchez ${ }^{\text {q }}$, M.P. Sanders ${ }^{\text {ad }}$,

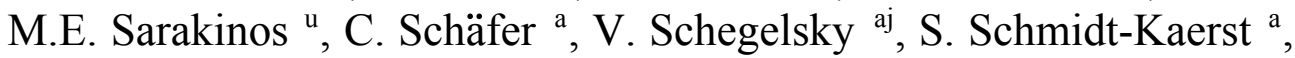
D. Schmitz ${ }^{\text {a }}$, H. Schopper ${ }^{\text {av }}$, D.J. Schotanus ${ }^{\text {ad }}$, G. Schwering ${ }^{\text {a }}$, 
C. Sciacca ${ }^{\text {ab }}$, D. Sciarrino ${ }^{\text {s }}$, A. Seganti i , L. Servoli af, S. Shevchenko ${ }^{\text {ae }}$, N. Shivarov an, V. Shoutko aa, E. Shumilov aa , A. Shvorob ae, T. Siedenburg a, D. Son ${ }^{\text {ao }}$, B. Smith ${ }^{\text {ag }}$, P. Spillantini ${ }^{\text {p }}$, M. Steuer ${ }^{n}$, D.P. Stickland ${ }^{\text {ah }}$, A. Stone ${ }^{\text {f }}$, H. Stone ${ }^{\text {ah, }, 3}$, B. Stoyanov ${ }^{\text {an }}$, A. Straessner ${ }^{\text {a }}$, K. Sudhakar ${ }^{\mathrm{j}}$, G. Sultanov ${ }^{\text {r }}$, L.Z. Sun ${ }^{\text {t, H. Suter }}{ }^{\text {au }}$, J.D. Swain ${ }^{\text {r }}$, Z. Szillasi ${ }^{\text {ap,4 }}$, T. Sztaricskai ${ }^{\text {ap, }}$, X.W. Tang ${ }^{\mathrm{g}}$, L. Tauscher ${ }^{\mathrm{e}}$, L. Taylor ${ }^{\mathrm{k}}$, C. Timmermans ${ }^{\mathrm{ad}}$, Samuel C.C. Ting ${ }^{\mathrm{n}}$, S.M. Ting ${ }^{n}$, S.C. Tonwar ${ }^{\mathrm{j}}$, J. Tóth ${ }^{\mathrm{m}}$, C. Tully ${ }^{\text {ah }}$, K.L. Tung ${ }^{\mathrm{g}}$, Y. Uchida ${ }^{\mathrm{n}}$, J. Ulbricht ${ }^{\text {au}}$, E. Valente ${ }^{\text {ai }}$, G. Vesztergombi ${ }^{\text {m}}$, I. Vetlitsky ${ }^{\text {aa }}$, D. Vicinanza ${ }^{\text {ak }}$, G. Viertel ${ }^{\text {au }}$, S. Villa ${ }^{\text {k }}$, M. Vivargent ${ }^{\mathrm{d}}$, S. Vlachos ${ }^{\text {e }}$, I. Vodopianov ${ }^{\text {aj, }}$, H. Vogel ${ }^{\text {ag }}$, H. Vogt ${ }^{\text {at }}$, I. Vorobiev ${ }^{\text {aa }}$, A.A. Vorobyov ${ }^{\text {aj }, ~ A . ~ V o r v o l a k o s ~}{ }^{\text {ac }}$, M. Wadhwa ${ }^{\mathrm{e}}$, W. Wallraff a , M. Wang ${ }^{\mathrm{n}}$, X.L. Wang ${ }^{\mathrm{t}}$, Z.M. Wang ${ }^{\mathrm{t}}$, A. Weber ${ }^{\mathrm{a}}$, M. Weber ${ }^{\text {a }}$, P. Wienemann ${ }^{\text {a }}$, H. Wilkens ${ }^{\text {ad }}$, S.X. Wu ${ }^{n}$, S. Wynhoff a ${ }^{\text {a }}$ L. Xia ${ }^{\text {ae }}$, Z.Z. Xu ${ }^{\mathrm{t}}$, B.Z. Yang ${ }^{\mathrm{t}}$, C.G. Yang ${ }^{\mathrm{g}}$, H.J. Yang ${ }^{\mathrm{g}}$, M. Yang ${ }^{\mathrm{g}}$, J.B. Ye ${ }^{\mathrm{t}}$, S.C. Yeh ${ }^{\mathrm{ax}}$, An. Zalite ${ }^{\text {aj }}$, Yu. Zalite ${ }^{\text {aj }}$, Z.P. Zhang ${ }^{\mathrm{t}}$, G.Y. Zhu ${ }^{\mathrm{g}}$, R.Y. Zhu ${ }^{\text {ae }}$, A. Zichichi i,q,r , F. Ziegler ${ }^{\text {at }}$, G. Zilizi ${ }^{\text {ap, } 4}$, M. Zöller ${ }^{\text {a }}$

${ }^{a}$ I. Physikalisches Institut, RWTH, D-52056 Aachen, Germany, and III. Physikalisches Institut, RWTH, D-52056 Aachen, Germany

${ }^{\mathrm{b}}$ National Institute for High Energy Physics, NIKHEF, and University of Amsterdam, NL-1009 DB Amsterdam, The Netherlands c University of Michigan, Ann Arbor, MI 48109, USA

${ }^{\mathrm{d}}$ Laboratoire d'Annecy-le-Vieux de Physique des Particules, LAPP, IN2P3-CNRS, BP 110, F-74941 Annecy-le-Vieux CEDEX, France

${ }^{\mathrm{e}}$ Institute of Physics, University of Basel, CH-4056 Basel, Switzerland

${ }^{\mathrm{f}}$ Louisiana State University, Baton Rouge, LA 70803, USA

${ }^{\mathrm{g}}$ Institute of High Energy Physics, IHEP, 100039 Beijing, China ${ }^{6}$

${ }^{\mathrm{h}}$ Humboldt University, D-10099 Berlin, Germany ${ }^{5}$

${ }^{\mathrm{i}}$ University of Bologna and INFN-Sezione di Bologna, I-40126 Bologna, Italy

${ }^{\mathrm{j}}$ Tata Institute of Fundamental Research, Bombay 400 005, India

${ }^{\mathrm{k}}$ Northeastern University, Boston, MA 02115, USA

${ }^{1}$ Institute of Atomic Physics and University of Bucharest, R-76900 Bucharest, Romania

${ }^{\mathrm{m}}$ Central Research Institute for Physics of the Hungarian Academy of Sciences, H-1525 Budapest 114, Hungary ${ }^{7}$

${ }^{\mathrm{n}}$ Massachusetts Institute of Technology, Cambridge, MA 02139, USA

${ }^{\circ}$ KLTE-ATOMKI, H-4010 Debrecen, Hungary ${ }^{4}$

${ }^{\mathrm{p}}$ INFN Sezione di Firenze and University of Florence, I-50125 Florence, Italy

${ }^{\mathrm{q}}$ European Laboratory for Particle Physics, CERN, CH-1211 Geneva 23, Switzerland

${ }^{\mathrm{r}}$ World Laboratory, FBLJA Project, CH-1211 Geneva 23, Switzerland

${ }^{\mathrm{s}}$ University of Geneva, CH-1211 Geneva 4, Switzerland

${ }^{\mathrm{t}}$ Chinese University of Science and Technology, USTC, Hefei, Anhui 230 029, China ${ }^{6}$

${ }^{u}$ SEFT, Research Institute for High Energy Physics, P.O. Box 9, SF-00014 Helsinki, Finland

${ }^{v}$ University of Lausanne, CH-1015 Lausanne, Switzerland

${ }^{\mathrm{w}}$ INFN-Sezione di Lecce and Universitá Degli Studi di Lecce, I-73100 Lecce, Italy

${ }^{x}$ Institut de Physique Nucléaire de Lyon, IN2P3-CNRS, Université Claude Bernard, F-69622 Villeurbanne, France

${ }^{y}$ Centro de Investigaciones Energéticas, Medioambientales y Tecnologícas, CIEMAT, E-28040 Madrid, Spain ${ }^{8}$

${ }^{\mathrm{z}}$ INFN-Sezione di Milano, I-20133 Milan, Italy

aa Institute of Theoretical and Experimental Physics, ITEP, Moscow, Russia

${ }^{\mathrm{ab}}$ INFN-Sezione di Napoli and University of Naples, I-80125 Naples, Italy

ac Department of Natural Sciences, University of Cyprus, Nicosia, Cyprus

${ }^{\text {ad }}$ University of Nijmegen and NIKHEF, NL-6525 ED Nijmegen, The Netherlands

${ }^{\text {ae }}$ California Institute of Technology, Pasadena, CA 91125, USA

af INFN-Sezione di Perugia and Universitá Degli Studi di Perugia, I-06100 Perugia, Italy

${ }^{\text {ag }}$ Carnegie Mellon University, Pittsburgh, PA 15213, USA

ah Princeton University, Princeton, NJ 08544, USA 


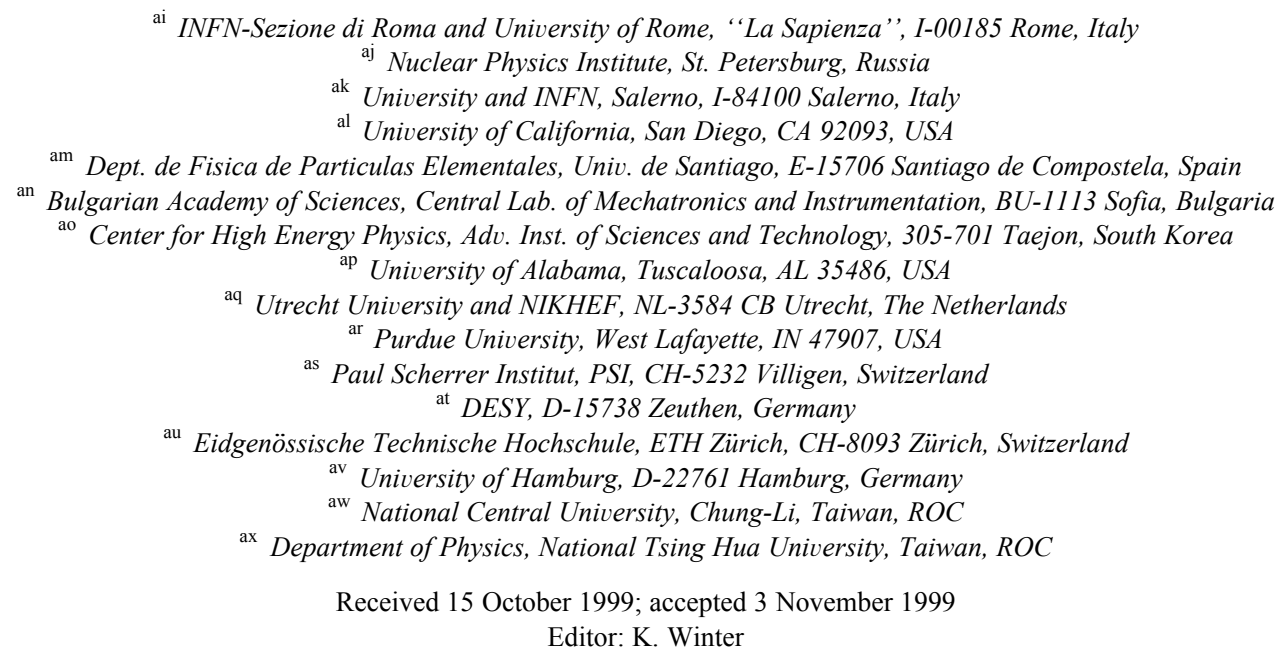

\section{Abstract}

Extra spatial dimensions are proposed by recent theories that postulate the scale of gravity to be of the same order as the electroweak scale. A sizeable interaction between gravitons and Standard Model particles is then predicted. Effects of these new interactions in boson and fermion pair production are searched for in the data sample collected at centre-of-mass energies above the $\mathrm{Z}$ pole by the $\mathrm{L} 3$ detector at LEP. In addition, the direct production of a graviton associated with a $\mathrm{Z}$ boson is investigated. No statistically significant hints for the existence of these effects are found and lower limits in excess of $1 \mathrm{TeV}$ are derived on the scale of this new theory of gravity. (c) 1999 Published by Elsevier Science B.V. All rights reserved.

\section{Introduction}

Contemporary collider experiments have successfully tested the Standard Model of electroweak interactions (SM) [1-3] at its characteristic distance $M_{\mathrm{ew}}^{-1}$, where $M_{\text {ew }} \sim 10^{2} \mathrm{GeV}$ represents the electroweak scale. The experimental study of the gravitational force extends only down to distances of the order of a centimetre [4], thirty three orders of magnitude above the distance $M_{\mathrm{Pl}}^{-1}$. The Planck scale $\left(M_{\mathrm{Pl}} \sim\right.$
$10^{19} \mathrm{GeV}$ ) denotes the characteristic scale of the gravitational interaction.

Deviations from the expected behaviour of gravity are expected in theories that introduce $n$ extra spatial dimensions of size $R$ that can be as large as a fraction of a millimetre [5]. This follows from postulating a new scale $M_{S}$ for the gravitational interaction, and imposing it to be of the same order of $M_{\mathrm{ew}}$. This Low Scale Gravity (LSG) is related to the macroscopic expectations of gravity in terms of the

\footnotetext{
${ }^{1}$ Also supported by CONICET and Universidad Nacional de La Plata, CC 67, 1900 La Plata, Argentina.

${ }^{2}$ Also supported by Panjab University, Chandigarh-160014, India.

${ }^{3}$ Deceased.

${ }^{4}$ Also supported by the Hungarian OTKA fund under contract numbers T22238 and T026178.

${ }^{5}$ Supported by the German Bundesministerium für Bildung, Wissenschaft, Forschung und Technologie.

${ }^{6}$ Supported by the National Natural Science Foundation of China.

${ }^{7}$ Supported by the Hungarian OTKA fund under contract numbers T019181, F023259 and T024011.

${ }^{8}$ Supported also by the Comisión Interministerial de Ciencia y Tecnología.
} 
gravitational constant and hence to $M_{\mathrm{Pl}}$ by the application of the Gauss' theorem in the extra dimensions:

$M_{\mathrm{Pl}}^{2} \sim R^{n} M_{S}^{n+2}$.

In the LSG scenario, spin-two gravitons couple with SM particles and contribute to the pair production of bosons and fermions in $\mathrm{e}^{+} \mathrm{e}^{-}$collisions. These effects were searched for previously [6]. The present analysis extends this investigation using the same procedure to the $176 \mathrm{pb}^{-1}$ of data collected by the L3 detector [7-13] at LEP in 1998 at the centreof-mass energy $\sqrt{s}=188.7 \mathrm{GeV}$. Other results are described in Ref. [14-17].

LSG effects in both boson and fermion pair production are described in terms of the parameter $M_{S}$ [18], interpreted as a cutoff of the theory. It appears as $1 / M_{S}^{4}$ in the LSG and SM interference terms and as $1 / M_{S}^{8}$ in the pure graviton exchange process. These terms are multiplied by the factors $\lambda$ and $\lambda^{2}$, respectively, which incorporate the dependence on the unknown full LSG theory and are of order unity [18]. For numerical results on the scale $M_{S}$, this analysis assumes $\lambda= \pm 1$ to allow for different signs in the interference between the SM and LSG contributions. Throughout this analysis the radiative corrections to SM and LSG processes are assumed to factorise.

LSG can also manifest itself via the direct production of a graviton associated with either a photon or a $\mathrm{Z}$ boson. The first of these signatures is investigated elsewhere $[6,19]$, while the second is reported here for the first time.

\section{Boson pair production}

The contribution of virtual graviton exchange to the pair production of $\mathrm{Z}$ bosons affects both the total cross section and the distribution of the $\mathrm{Z}$ production angle [20]. The same discriminating variables previously used to measure the $\mathrm{ZZ}$ cross section and to limit a possible $Z Z \gamma$ or ZZZ vertex [21] are investigated to search for LSG effects, namely the reconstructed $\mathrm{Z}$ mass of $\mathrm{ZZ} \rightarrow \mathrm{q} \overline{\mathrm{q}} \ell^{+} \ell^{-}$events, $M_{\mathrm{Z}}$, the output of a neural network for the $Z Z \rightarrow q \bar{q} q^{\prime} \bar{q}^{\prime}$ and $\mathrm{ZZ} \rightarrow \mathrm{q} \overline{\mathrm{q}} \nu \bar{v}$ final states and the sum of the visible and recoil masses in the $\mathrm{ZZ} \rightarrow \ell^{+} \ell^{-} \ell^{\prime+} \ell^{--}$and
$\mathrm{ZZ} \rightarrow \ell^{+} \ell^{-} \nu \bar{v}$ channels. $\mathrm{ZZ}$ events are generated with the EXCALIBUR Monte Carlo (MC) program [22]. LSG effects are modelled by reweighting these events with a modified version of EXCALIBUR that includes the LSG matrix element for the $\mathrm{ZZ}$ final state [20]. Fig. 1a presents the distributions of $M_{Z}$ for data, SM expectations and LSG predictions.

LSG effects in $\mathrm{W}$ pair production modify its differential cross section [20], as displayed in Fig. 1b and $1 \mathrm{c}$, where the polar angle of the emitted $\mathrm{W}^{-}$ boson is shown. Semileptonic and hadronic decays of the W pairs are analysed [23]. Data and SM expectations as calculated by the KORALW MC [24] are also illustrated. The inclusion of LSG effects proceeds through the reweighting of the MC events with a modified version of EXCALIBUR [25] which includes the virtual graviton exchange matrix element [20] for double-resonant processes. A 5\% cor-
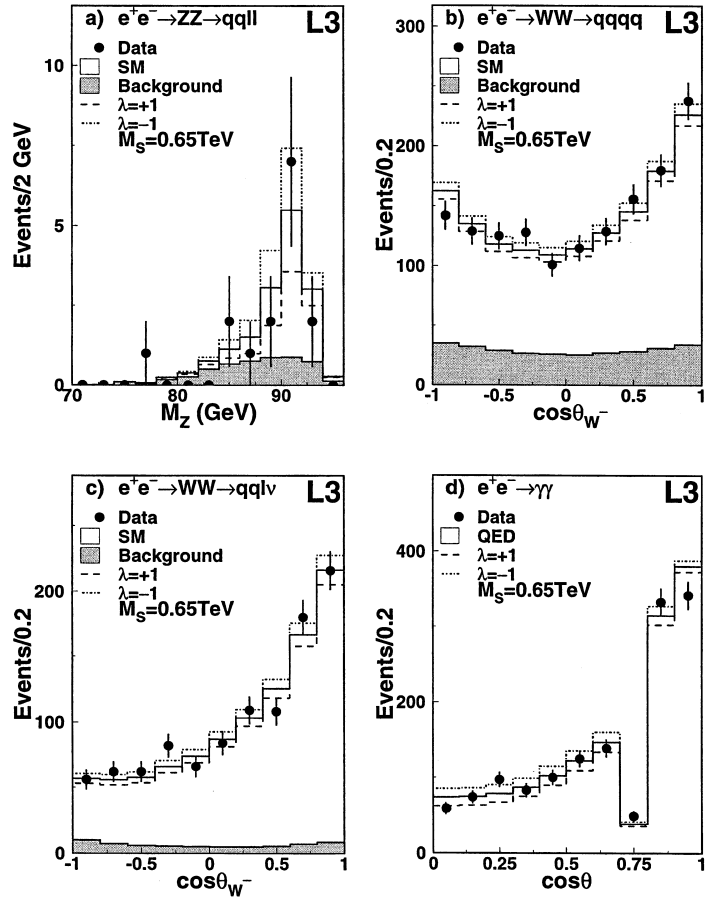

Fig. 1. (a) Reconstructed $\mathrm{Z}$ mass for $\mathrm{e}^{+} \mathrm{e}^{-} \rightarrow \mathrm{ZZ} \rightarrow \mathrm{q} \overline{\mathrm{q}} \ell^{+} \ell^{-}$ events. Distributions of the polar angle for: (b) hadronic $\mathrm{e}^{+} \mathrm{e}^{-} \rightarrow$ $\mathrm{W}^{+} \mathrm{W}^{-}$events, (c) semileptonic $\mathrm{e}^{+} \mathrm{e}^{-} \rightarrow \mathrm{W}^{+} \mathrm{W}^{-}$events, (d) $\mathrm{e}^{+} \mathrm{e}^{-} \rightarrow \gamma \gamma$ events. Data at $188.7 \mathrm{GeV}, \mathrm{SM}$ signal and background expectations are presented together with LSG predictions for $M_{S}=0.65 \mathrm{TeV}$ and $\lambda= \pm 1$. 
rection is applied to account for other diagrams contributing to the semileptonic electron final states.

The differential cross section of the process $\mathrm{e}^{+} \mathrm{e}^{-}$ $\rightarrow \gamma \gamma$ is also sensitive to $s$-channel graviton exchange [26,20]. Fig. 1d compares the photon polar angle distribution of the data [27] to LSG and QED predictions.

\section{Fermion pair production}

Contrary to boson pair production, where effects of extra dimensions are mainly expected in the total cross section, in the case of fermion pair production distortions of the angular distributions occur [18,26]. Events with high effective centre-of-mass energy, $\sqrt{s^{\prime}},\left(\sqrt{s^{\prime}}>0.85 \sqrt{s}\right)$ are studied.

Fig. $2 \mathrm{a}$ and $2 \mathrm{~b}$ compare the angular distributions of muon and tau pairs selected in data [28] to SM and LSG expectations. The SM predictions are modelled by MC events generated with KORALZ [29] and reweighted to the ZFITTER [30] differential cross sections. The effects of extra dimensions are studied by reweighting these events to the LSG differential cross sections $[18,26]$.

For the $\mathrm{q} \overline{\mathrm{q}}$ final states only the total cross section is investigated and thus the higher sensitivity interference term $[18,26]$ vanishes. The measurement of the cross section [28] is sensitive only to the pure graviton exchange and is independent of the sign of $\lambda$.
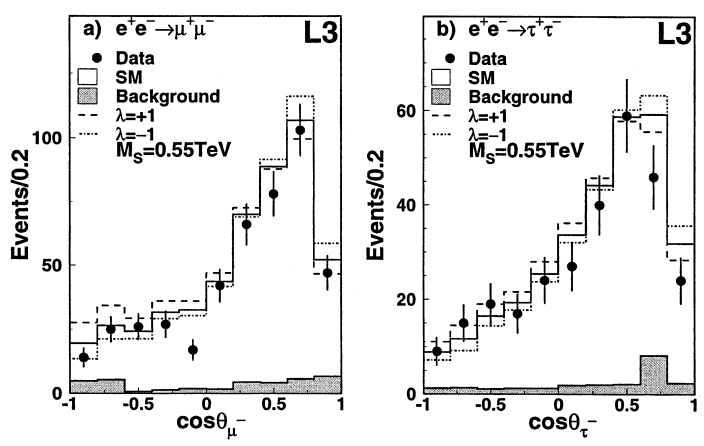

Fig. 2. Distributions of the fermion polar angle for: (a) $\mathrm{e}^{+} \mathrm{e}^{-} \rightarrow$ $\mu^{+} \mu^{-}$and (b) $\mathrm{e}^{+} \mathrm{e}^{-} \rightarrow \tau^{+} \tau^{-}$processes. Selected data events at $188.7 \mathrm{GeV}$ are shown together with SM signal and background expectations. LSG predictions for the two signs of the interference and $M_{S}=0.55 \mathrm{TeV}$ are also presented.

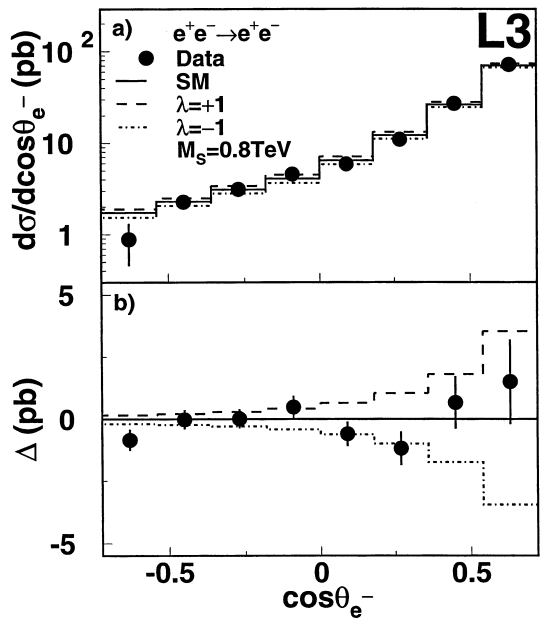

Fig. 3. a) Measured and predicted differential cross sections for Bhabha scattering. LSG expectations for $\lambda= \pm 1$ and $M_{S}=$ $0.8 \mathrm{TeV}$ are also shown. b) Differences $\Delta$ of the measured and LSG differential cross sections with respect to the SM prediction. Data collected at $188.7 \mathrm{GeV}$ are presented.

The Bhabha scattering is the channel with the highest sensitivity to LSG effects owing to their large interference with the SM $t$-channel diagram. Fig. 3 presents the measured differential cross section for events in the polar angular range of the scattered electron between $44^{\circ}$ and $136^{\circ}$. The SM predictions as obtained from TOPAZ0 [31] are also shown together with the deviations expected from LSG [32].

\section{Results}

The distributions in Figs. 1-3a are separately analysed in terms of $\lambda / M_{S}^{4}$. In addition the $\mathrm{q} \overline{\mathrm{q}}$ cross section is compared with the LSG predictions in terms of $\lambda^{2} / M_{S}^{8}$ and the other four distributions describing the different decay modes of the $\mathrm{Z}$ boson pair production [21] are considered as well. Previous results from lower $\sqrt{s}$ data [6] are included. A likelihood is determined as a function of $\lambda / M_{S}^{4}$. The deviation $\delta$ of the maximum of the likelihood from the SM value of zero is measured in units of one sigma errors and is reported in Table 1 . In all the quoted fits, the background dependence on LSG 
effects is negligible. No significant deviations from the SM expectations are found. The likelihood functions are then integrated over the physical region for $\lambda=+1$ and $\lambda=-1$ to yield the $95 \%$ confidence level (CL) limits on $M_{S}$, also reported in Table 1.

The sensitivity of the $\mathrm{ZZ}$ channel is comparable to that of the $\gamma \gamma$ and $\mathrm{W}^{+} \mathrm{W}^{-}$ones, despite the significantly lower cross section. The expected high sensitivity of the Bhabha channel is confirmed, as it dominates the limits.

Systematic uncertainties are included in the fit. They are calculated as the sum in quadrature of the systematic error on the measured cross section and the theory uncertainty on its prediction and amount to $10 \%$ for the $\mathrm{ZZ}$ channel, $4 \%$ for $\mathrm{WW}, 1 \%$ for $\gamma \gamma$, $2.4 \%$ for $\mu^{+} \mu^{-}, 3.5 \%$ for $\tau^{+} \tau^{-}, 1.4 \%$ for $\mathrm{q} \overline{\mathrm{q}}$ and $3.0 \%$ for Bhabha scattering. Only the limits from the $\mathrm{q} \overline{\mathrm{q}}$ and Bhabha channels are affected by these systematic effects.

Assuming that no higher order operators give sizeable contributions to the LSG mediated boson and fermion pair production and that the meaning of the cutoff parameter is the same for all the investigated processes, it is possible to fit simultaneously all the boson and fermion channels, and finally to combine these two results into a final fit. No statistically significant extra dimensions effects are found. The 95\% CL lower limits on $M_{S}$ are listed in Table

Table 1

Lower limits at $95 \% \mathrm{CL}$ on the cutoff $M_{S}$ for different processes and values of $\lambda$. Deviations $\delta$ from the SM, defined in the text, are also given

\begin{tabular}{cccc}
\hline Process & $\delta$ & $\begin{array}{c}M_{S}(\mathrm{TeV}) \\
\lambda=+1\end{array}$ & $\begin{array}{c}M_{S}(\mathrm{TeV}) \\
\lambda=-1\end{array}$ \\
\hline $\mathrm{e}^{+} \mathrm{e}^{-} \rightarrow \mathrm{ZZ}$ & -0.55 & 0.77 & 0.76 \\
$\mathrm{e}^{+} \mathrm{e}^{-} \rightarrow \mathrm{W}^{+} \mathrm{W}^{-}$ & -1.10 & 0.79 & 0.68 \\
$\mathrm{e}^{+} \mathrm{e}^{-} \rightarrow \gamma \gamma$ & -0.03 & 0.79 & 0.80 \\
Bosons combined & -0.79 & 0.89 & 0.82 \\
$\mathrm{e}^{+} \mathrm{e}^{-} \rightarrow \mu^{+} \mu^{-}$ & -1.12 & 0.69 & 0.56 \\
$\mathrm{e}^{+} \mathrm{e}^{-} \rightarrow \tau^{+} \tau^{-}$ & +0.56 & 0.54 & 0.58 \\
$\mathrm{e}^{+} \mathrm{e}^{-} \rightarrow \mathrm{q} \overline{\mathrm{q}}$ & \pm 2.30 & 0.49 & 0.49 \\
$\mathrm{e}^{+} \mathrm{e}^{-} \rightarrow \mathrm{e}^{+} \mathrm{e}^{-}$ & -0.91 & 0.98 & 0.84 \\
Fermions combined & -1.04 & 1.00 & 0.84 \\
Bosons + Fermions & -1.30 & 1.07 & 0.87 \\
\hline
\end{tabular}

Table 2

Expected cross sections $\sigma_{\mathrm{ZG}}$ for the graviton plus $\mathrm{Z}$ signal $\left(M_{S}=0.5 \mathrm{TeV}\right)$, detection efficiency $\varepsilon$, upper limit at $95 \% \mathrm{CL}$ $\sigma_{\mathrm{ZG}}^{\lim }$ on the cross section and lower limit on the scale $M_{S}$ as a function of the number of extra dimensions $n$

\begin{tabular}{llll}
\hline$n$ & 2 & 3 & 4 \\
\hline$\sigma_{\mathrm{ZG}}(\mathrm{pb})$ & 0.64 & 0.081 & 0.011 \\
$\varepsilon$ & 0.56 & 0.56 & 0.55 \\
$\sigma_{\mathrm{ZG}}^{\lim }(\mathrm{pb})$ & 0.29 & 0.30 & 0.30 \\
$M_{S}(\mathrm{TeV})$ & 0.60 & 0.38 & 0.29 \\
\hline
\end{tabular}

1. They reach $1.07 \mathrm{TeV}$ for $\lambda=+1$ and $0.87 \mathrm{TeV}$ for $\lambda=-1$.

\section{Single $Z$ production}

In addition to the possible LSG effects in pair production of SM particles, the direct graviton $(\mathrm{G})$ production associated with a $\mathrm{Z}$ boson is studied for the first time, complementing the $G \gamma$ search $[6,19]$. The expected cross section $\sigma_{\mathrm{ZG}}$ is proportional to $M_{S}^{-(n+2)}$ [33] and thus falls rapidly with the number of extra dimensions $n$. It is shown in Table 2 for a benchmark value of $M_{S}=0.5 \mathrm{TeV}$. The symbol $M_{S}$ represents the LSG scale analogous to the $M_{D}$ pa-

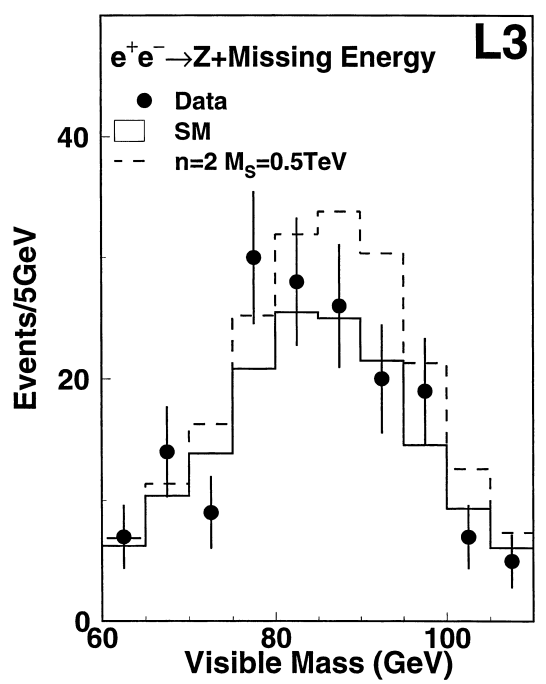

Fig. 4. Visible mass for $\mathrm{e}^{+} \mathrm{e}^{-} \rightarrow \mathrm{Z} G$ candidate events at 188.7 GeV together with SM expectations, dominated by W pair and single $\mathrm{W}$ production. The effect of real graviton production with two extra space dimensions and $M_{S}=0.5 \mathrm{TeV}$ is also shown. 
rameter [26] investigated in the $\mathrm{G} \gamma$ channel. In the particular case of $n=2$ the two parameters are related by $M_{S}^{4}=4 M_{D}^{4}$ [34]. The reduced sensitivity with respect to the $G \gamma$ channel follows from the limited phase space available for graviton emission due to the mass of the $Z$.

The signature of this process is a single $\mathrm{Z}$ boson in the detector as the graviton is emitted in the extra dimensions and hence undetected. Only hadronic $\mathrm{Z}$ decays are considered. A sample of 1068 unbalanced hadronic events with missing energy pointing in the detector and a visible mass compatible with that of the $\mathrm{Z}$ is selected. The SM expectation amounts to 1096 events. The signal efficiency is $87 \%$. Efficiencies and distributions for the signal are estimated by analysing a sample of $\mathrm{e}^{+} \mathrm{e}^{-} \rightarrow \mathrm{Z} \nu \bar{\nu}$ events generated in the $\mathrm{W}$ fusion process with EXCALIBUR and then reweighted to the $\mathrm{Z}$ energy and polar angle distributions of the $\mathrm{e}^{+} \mathrm{e}^{-} \rightarrow Z G$ process [33]. The analysis is designed to be independent of $n$.

Event-shape and jet-shape variables similar to those used in the $\mathrm{e}^{+} \mathrm{e}^{-} \rightarrow \mathrm{ZZ} \rightarrow \mathrm{q} \overline{\mathrm{q}} \nu \bar{\nu}$ [21] and hadronicaly decaying single $\mathrm{W}$ [35] selections are used to suppress the dominant backgrounds: radiative return to the $\mathrm{Z}$, double resonant $\mathrm{W}$ pair production followed by semileptonic decays into either a tau or an undetected low angle lepton and hadronic decaying single $\mathrm{W}$ events. A final sample of 129 events is selected with 126 expected from SM processes with the signal efficiencies listed in Table 2. A fit to the visible mass distribution of Fig. 4 yields the $95 \%$ CL cross section upper limits in Table 2 from which the corresponding lower limits on $M_{S}$ are extracted.

In conclusion no evidence for extra dimensions is found and limits in excess of $1 \mathrm{TeV}$ are set on the scale of LSG.

\section{Acknowledgements}

We thank Kingman Cheung for useful discussions on the associated $\mathrm{Z}$ and graviton production. We wish to express our gratitude to the CERN accelerator divisions for the superb performance and the continuous and successful upgrade of the LEP machine. We acknowledge the contributions of the engineers and technicians who have participated in the construction and maintenance of this experiment.

\section{References}

[1] S.L. Glashow, Nucl. Phys. 22 (1961) 579

[2] A. Salam, in: N. Svartholm (Ed.), Elementary Particle Theory, Almqvist and Wiksell, Stockholm, 1968, p. 367.

[3] S. Weinberg, Phys. Rev. Lett. 19 (1967) 1264.

[4] J.C. Long et al., Nucl. Phys. B 539 (1999) 23.

[5] N. Arkani-Hamed et al., Phys. Lett. B 429 (1998) 263.

[6] L3 Collaboration, M. Acciarri et al., preprint CERN-EP /99117, hep-ex/9909019.

[7] L3 Collaboration, B. Adeva et al., Nucl. Inst. Meth. A 289 (1990) 35 .

[8] L3 Collaboration, O. Adriani et al., Phys. Rep. 236 (1993) 1.

[9] I.C. Brock et al., Nucl. Instr. and Meth. A 381 (1996) 236.

[10] M. Chemarin et al., Nucl. Inst. Meth. A 349 (1994) 345.

[11] M. Acciarri et al., Nucl. Inst. Meth. A 351 (1994) 300.

[12] A. Adam et al., Nucl. Inst. Meth. A 383 (1996) 342.

[13] G. Basti et al., Nucl. Inst. Meth. A 374 (1996) 293.

[14] OPAL Collaboration, G. Abbiendi et al., preprint CERNEP/99-088, hep-ex/9907064.

[15] OPAL Collaboration, G. Abbiendi et al., preprint CERNEP/99-097, hep-ex/9908008.

[16] S. Mele, E. Sanchez, preprint CERN-EP/99-118, hep$\mathrm{ph} / 9909294$.

[17] D. Bourilkov, J. High Energy Phys. 08 (1999) 006.

[18] J. Hewett, Phys. Rev. Lett. 82 (1999) 4765.

[19] L3 Collaboration, M. Acciarri et al., preprint CERN-EP/99129, hep-ex/9910009.

[20] K. Agashe, N.G. Deshpande, Phys. Lett. B 456 (1999) 60.

[21] L3 Collaboration, M. Acciarri et al., preprint CERN-EP/99119, hep-ex/9909043.

[22] R. Kleiss, R. Pittau, Comp. Phys. Comm. 83 (1994) 141; R. Pittau, Phys. Lett. B 335 (1994) 490.

[23] L3 Collaboration, M. Acciarri et al., contributed paper \#6 254 to the EPS conference, Tampere, Finland 1999; paper in preparation.

[24] M. Skrzypek et al., Comp. Phys. Comm. 94 (1996) 216; Phys. Lett. B 372 (1996) 289.

[25] R. Pittau, private communication.

[26] G.F. Giudice et al., Nucl. Phys. B 544 (1999) 3.

[27] L3 Collaboration, M. Acciarri et al., contributed paper \#6 251 to the EPS conference, Tampere, Finland 1999; paper in preparation.

[28] L3 Collaboration, M. Acciarri et al, contributed paper \#6 262 to the EPS conference, Tampere, Finland 1999; paper in preparation.

[29] S. Jadach et al., Comp. Phys. Comm. 79 (1994) 503.

[30] D. Bardin et al., Preprint CERN-TH/6443/92; Z. Phys. C 44 (1989) 493; Nucl. Phys. B 351 (1991) 1; Phys. Lett. B 255 (1991) 290.

[31] G. Montagna et al., Nucl. Phys. B 401 (1993) 3; Comp. Phys. Comm. 76 (1993) 238.

[32] T. Rizzo, Phys. Rev. D 59 (1999) 115010.

[33] K. Cheung, W.-Y. Keung, hep-ph/9903294.

[34] Kingman Cheung, private communication.

[35] L3 Collaboration, M. Acciarri et al., Phys. Lett. B 436 (1998) 417. 